\title{
Contemporary principles of diagnostic and therapeutic management in cervical and ovarian neuroendocrine tumors
}

\author{
Mariusz Bidzinski ${ }^{1}\left(\mathbb{D}\right.$, Szymon Piatek ${ }^{1} \mathbb{D}$, Jaroslaw Cwikla ${ }^{2}(\mathbb{D}$, \\ Anna Nasierowska-Guttmejer ${ }^{3,4}$, Anna Danska-Bidzinska ${ }^{5}$ (i) \\ ${ }^{1}$ Department of Gynecologic Oncology, Maria Sklodowska-Curie National Research Institute of Oncology Warsaw, Poland \\ ${ }^{2}$ Department of Radiology, University of Warmia and Mazury, Olsztyn, Poland \\ ${ }^{3}$ Pathology Department, Central Clinical Hospital of the MSWiA, Warsaw, Poland \\ ${ }^{4}$ Lazarski University, Warsaw, Poland \\ ${ }^{5} 2^{\text {nd }}$ Clinic of Obstetrics and Gynecology, Medical University of Warsaw, Poland
}

\begin{abstract}
Enhancing knowledge about neuroendocrine neoplasms causes the need to improve management of these tumors. Although these tumors are rare in clinical practice, their biological diversity makes both diagnostics and therapy a challenge for contemporary oncology. The article discusses the latest developments in the diagnostic procedures and methods of treatment of the cervical and ovarian neuroendocrine tumors. Algorithms are presented to understand the differences in therapeutic management in these malignancies.

Key words: cervical neuroendocrine neoplasm; ovarian neuroendocrine neoplasm; carcinoid; management; small cell hypercalcemic ovarian tumor
\end{abstract}

Ginekologia Polska 2021; 92, 4: 312-317

\section{INTRODUCTION}

Primary neuroendocrine neoplasms/tumors (NEN/NETs) of female genital organs require a separate approach due to extremely aggressive course. These tumors present as heterogeneous group with an incidence of about two percent of all female genital cancers [1, 2]. The cervix and ovaries are the most common locations of neuroendocrine tumors within the female reproductive system; only isolated cases have been described in the uterine corpus. NEN metastases from the gastrointestinal tract, lungs and thymus should be always excluded, especially in ovaries involvement. These tumors require an appropriate histopathological assessment and should be distinguished from other primary ovarian neoplasms, particularly granulosa cell tumor.

The terminology of neoplasm, commonly called carcinoid, has been changed since 2000 [3]. European Neuroendocrine Tumor Society, due to new opportunities in somatostatin receptor-targeted therapy within the neoplastic cells, distinguished two basic groups in NEN: low-grade neuroendocrine neoplasms/tumors (LG NENs/NETs) microscopically resembling carcinoids, and high-grade neuroendocrine neoplasms/tumors ( HG NENs/NETs) with cancer morphology.

\section{CERVICAL NENS}

Nowadays, according to theWHO classification from 2014, cervical LG NENs are divided into the following groups [4]:

- low grade neuroendocrine tumors (TC, carcinoid tumor),

- low grade neuroendocrine tumors, G1

- low grade neuroendocrine tumors, (AC, atypical carcinoid tumor),

- low grade neuroendocrine tumors, G2.

Cervical HG NENs referred to as neuroendocrine carcinoma G3 (NEC) should be classified as tumors of the digestive system. The WHO classification distinguishes small cell neuroendocrine carcinoma (SCNEC) and large cell neuroendocrine carcinoma (LCNEC).

\section{Cytological diagnostics}

Pap smears are of little use in detecting early forms of the cervical NEN. Only for an experienced cytopathologist

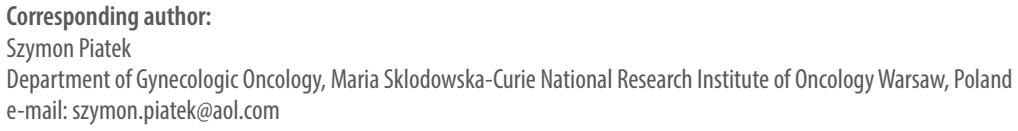


or pathologist, the cytological test raises suspicion of NEN. Chiang et al. recorded $57.5 \%$ normal smear in a group of 133 patients with SCNEC. The authors found that false negative results were significantly higher compared to squamous cell cervical cancer [5]. In another study, abnormal cytology was detected in $14-45.5 \%$ of cases [6]. Zhou et al. [7] presented an analysis of cytological smears from 11 patients with cervical NEN. In six cases, abnormal cells were found in three patients non-specific cancer cells, in another three patients adenocarcinoma cells were diagnosed. Retrospective evaluation of seven smears, assessed initially as normal, showed abnormal cells in two patients. These cases were found to be false negative. Park et al. [8] observed abnormal cytology in 9 of 27 patients (33.33\%) with cervical NEN. In two cases neuroendocrine tumor cells were detected in cytological smear; the others were diagnosed with squamous cell carcinoma ( $n=2)$, HSIL $(n=3)$, ASCUS $(n=2)$. Kim et al. [9] attempted to determine the characteristic smear features for cervical SCNEC. They analyzed 13 cases of cervical SCNEC and compared them with squamous cell carcinoma, lymphoma and chronic cervicitis. Cytological changes useful in differential diagnosis were found: nuclear molding and smearing $(100 \%)$, salt and pepper chromatin (100\%), exudative and necrotic background (91.7\%), various architectures including individual cells (83.3\%), tight clusters (75\%) and feathering and strip (50\%), and inconspicuous nucleoli (75\%).

Nonetheless cervical Pap smear test is insufficient for the diagnosis of NEN.

\section{Histopathological and molecular diagnostics}

Macroscopically cervical NENs are not different from squamous cell and adenocarcinoma. Diagnosis is based on a histopathological examination with immunohistochemical assay. Cervical NENs differentiate from squamous and glandular epithelium. Neuroendocrine tumors, especially low grade can produce various proteins and hormones like calcitonin, gastrin, serotonin, substance $P$, vasoactive intestinal peptide, pancreatic polypeptide, somatostatin and adrenocorticotrophic hormone. Nonetheless cervical NENs rarely demonstrate clinical symptoms. Carcinoid syndrome can be found in cases with liver metastases.

Microscopically LG NENs are characterized by organoid structures resembling carcinoids. Accordingly, to cell maturity they are divided into two subtypes [10]. LG NEN (G1) differs from LG NEN G2 in nuclear atypia, mitotic activity and the presence of focal thrombotic necrosis. Proliferation activity of Ki-67, which has significant predictive and prognostic value in the NEN of the digestive system, is not of that importance in the NEN of the cervix. Cervical HG NEC $\mathrm{G} 3$ resembles small- and large-cell lung cancers as far as microscopic image is concerned. They are characterized by high proliferation activity, extensive infiltration and necrosis.
Diagnosis of NEN requires confirmation of immunohistochemical (IHC) markers. Chromogranin A and synaptophysin are mandatory in every case. The expression of other markers like neuron- specific enolase (NSE) and CD56 may also be useful [4]. These markers are specific in 33 to $100 \%$ of small cell neuroendocrine tumors [10]. In some cases, particular in SCNENs, IHC expression of these markers may be weak.

Recently, Japanese researchers pointed out that insulinoma associated protein 1 (INSM 1) is more specific than other markers [11]. In addition, thyroid transcription factor 1 (TTF1) was found to be specific in SCNET in 33\% to $84 \%$ [12]. Usefulness of TTF1 is limited, because it did not allow to distinguish SCNET from primary pulmonary NEN. Due to frequent coexistence of cervical NENs and HPV infection (especially high-risk 18 type) a positive reaction to $\mathrm{p} 16$ protein can be found. In the meta-analysis published by Castle et al. [13] HPV 16 and/or 18 infections was found in $85 \%$ of SCNENs and $88 \%$ of LCNENs. The activity of Ki-67 in cervical NETs is not obvious as in gastrointestinal tract and lung NENs. Moreover, in WHO classification 2014, the Ki-67 index was not included in the diagnostic criteria of cervical neuroendocrine tumors [4]. Among molecular abnormalities, mutations in the following genes are most common: c-myc (53\%), p53 (26\%), PIK3CA (18\%). Loss of heterozygosity was found in approximately $30 \%$ of NETs of female reproductive system [10].

\section{Imaging diagnostics}

Chest, abdominal and pelvic computed tomography (CT) should be performed in each case of cervical NET. PET $\mathrm{CT}$ is also recommended. Pelvic MRI or a transrectal ultrasound (TRUSG) examination should be additionally performed in locally advanced disease. Accuracy of parametria involvement in tumors $<1 \mathrm{~cm}$ compared with the histopathological examination was $98.7 \%$ and $94.7 \%(p<0.219)$, respectively [14]. MRI is the method of choice for staging cervical NETs in pregnant women, in cases of iodine contrast allergy or renal failure [15].

Somatostatin receptor scintigraphy (SRS) can be helpful in appropriate staging or searching for the primary lesion in well differentiated NENs due to overexpression of the somatostatin (SST) receptor. Other cervical NENs do not have significant expression of SST receptors. In these cases, positron emission tomography with fluorodeoxyglucose (FDG-PET) is an option. FDG-PET is used in staging and has prognostic significance in low differentiation NENs. It can also be used in identification of the primary tumor, assessment of treatment effectiveness or suspicion of relapse [16].

\section{Clinical course, prognosis and treatment LG NENs G1 and LG NENs G2}

They account for 0.5 to $5 \%$ of all cervical cancers [17]. Primary LG NEN G1 is seldomly diagnosed. The metastatic 
character of the tumor should always be excluded. The most common clinical symptom is vaginal bleeding. Symptoms of carcinoid syndrome are rarely manifested in these patients, although it is often possible to detect serum elevated concentrations of 5-hydroxyindoleacetic acid (5-HIAA). The clinical course of LG NEN G1 is difficult to predict due to its rare occurrence. LG NEN G2 is an extremely aggressive tumor. Between two to three-years overall survival range between 12.5 and 33\%. Even early stage tumors may spread to distant locations through lymphatic drainage or blood. The treatment of LG NEN G2 is not standardized although total abdominal hysterectomy is proposed in a locally advanced tumor. Isolated liver metastases may be treated with transarterial chemoembolization (TACE) using streptozotocin and 5-fluorouracyl. It is more effective than systemic treatment with paclitaxel, cis- or carboplatin, which have been shown to be ineffective in the treatment of LG NEN G2 metastases. In cases of positive somatostatin receptor, treatment with somatostatin analogues can be used $[10,18]$.

\section{HG NENs G3}

The average age of patients with SCNEN ranges from 37 to 46 years. Lymphatic or blood metastases occur immediately even in early stages of disease. Perineural invasion is common. Staging is the most important prognostic factor. Postoperative histopathological examination revealed metastases in lymph nodes in $45-57 \%$ of cases and LVSI involvement in $80 \%$ of cases $[19,20]$. The mean overall survival in stage I-IIA is 31 months, while in stage IIB - IVB it reaches 10 months. According to SEER data, five-years survival in early stages ranges from 30 to $60 \%$, and in advanced stages is $0-17 \%$ [21]. The results of treatment are significantly worse than in other types of the cervical cancer. Five-year survival in SCNENs, squamous cell carcinoma and adenocarcinoma are $35.7 \%, 60.5 \%$ and $69.7 \%$ respectively [22]. Tumor size is also prognostic. Five-year survival in tumors $\leq 4 \mathrm{~cm}$ and $>4 \mathrm{~cm}$ was $76 \%$ and $18 \%$, respectively.

As in other rare cancers, there is a lack of data on treatment based on randomized trials. The experience gathered during treatment of pulmonary NENs is used in therapy of gynecological NENs. At the moment of diagnosis of SCNEN metastases are often found, so treatment is usually multidisciplinary.

Incidence of LCNEN is less than SCNEN but it also has aggressive clinical course. Most patients with LCNEN die within the first three years since diagnosis. Embry et al. [23] analysed 63 patients with LCNENs and found that the average survival was 16.5 months (19 months - stage I, 17 months - stage II, 3 months - stage III, 1.5 moths - stage IV). Weak HER-2/Neu expression and strong EGFR expression appeared to be adverse prognostic factors. MD Anderson Cancer Center (MDACC) way of treatment of LCNENs is shown in Figure 1 [24]. MDACC is currently one of the leading institutions in developing diagnostic and treatment strategy of NENs of female reproductive system. Alternative management was shown by Gadducci et al. [19]. Their therapeutic chart is presented on Figure 2.

\section{OVARIAN NENS}

According to WHO 2014 classification ovarian neuroendocrine tumors are divided into two groups [4]. The first

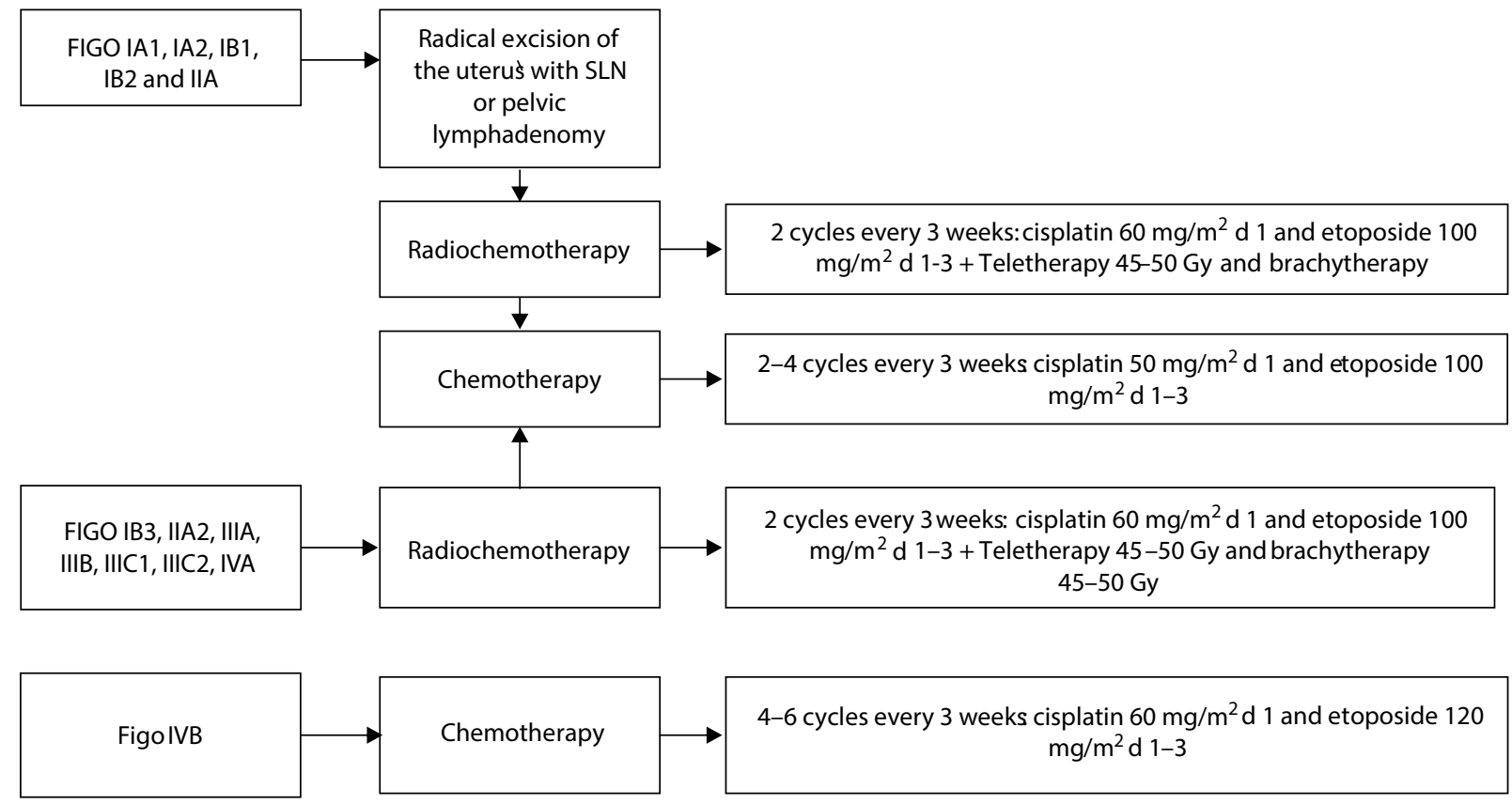

Figure 1. A therapeutic regimen in high grade cervical NETs adopted with recommendation of the MD Anderson Cancer Center 

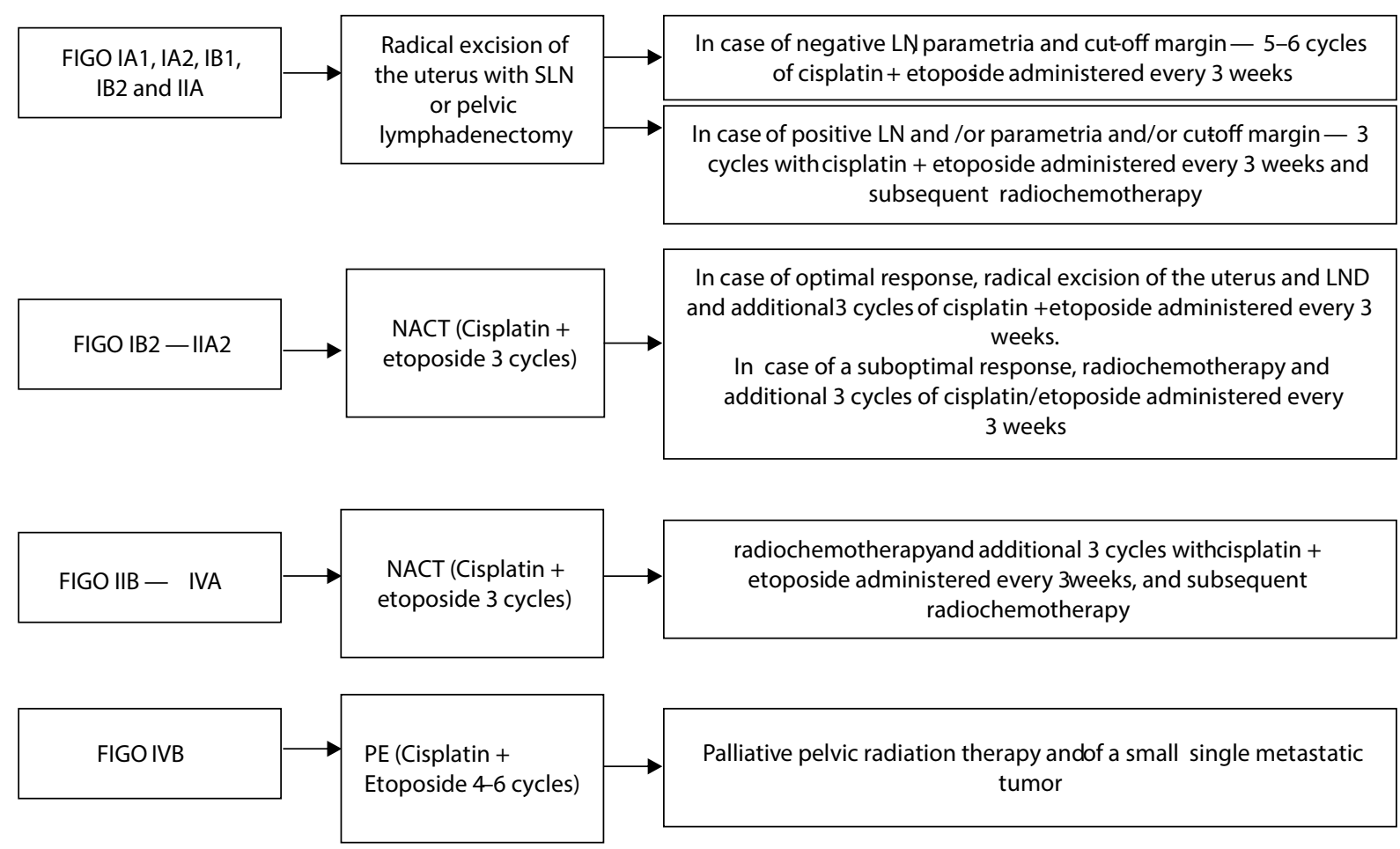

Figure 2. Therapeutic procedure scheme in high grade cervical NETs developed by Gadducci

group consists of low-grade neuroendocrine neoplasms resembling NEN of gastrointestinal system. They are identified as carcinoid (Carcinoid/Tumor carcinoid - TC) and correspond to low grade neuroendocrine tumors $\mathrm{G} 1$ (well differentiated neuroendocrine tumor/ low grade neuroendocrine tumor). The second category of ovarian NENs are poorly differentiated tumors (high grade NETs, HG NETs). The following types are found among HG NETs:

1. small cell carcinoma, hypercalcemic type (SCCHT),

2. small cell carcinoma, pulmonary type ( SCCPT),

3. large cell neuroendocrine carcinoma (LCNEC).

\section{LG NENS}

Carcinoids (TC) are the most common ovarian NENs. They account for only five percent of all carcinoids and represent only $0.1 \%$ of all malignant ovarian neoplasms [17]. There are three types of TC:

a) a component of a mature teratoma

b) primary ovarian carcinoid

c) metastatic carcinoid

TC forming as a component of mature teratoma constitutes about $75 \%$ cases of ovarian carcinoids. It is usually found as unilateral lesion, but even in $15 \%$ of cases, mature cystic teratoma, mucinous tumor or Brenner tumor may be found in opposite site. Symptoms of carcinoid syndrome may occur in about $1 / 4$ to $1 / 3$ of cases. In the WHO classification 2014, ovarian carcinoids are included in the germinal ovarian tumors as teratoma with possibility of secretion of neuroendocrine substances that may cause carcinoid syndrome. Serotonin-derived molecule is secreted directly into the ovarian vein, bypasses the hepatic passage, and causes symptoms such as hot flushes, diarrheas, bronchospasm or edemas. Y peptide disturbs intestinal motility and leads to constipations. Other substances include pancreatic polypeptide, gastrin or glucagon [1,25]. The average age at the moment of diagnosis TC is 55 years; most patients are women in perimenopause or menopause.

Primary ovarian carcinoid occurs in four microscopic types: insular, trabecular, mucinous and stromal. The insular type resembles neuroendocrine tumors of the middle segment of the archenteron, while the trabecular type imitates neuroendocrine neoplasms from the anterior and posterior sections of the archenteron. The mitotic figures are rare. Microscopic structure of the mucinous variant is akin to an appendix carcinoid and three histologic subtypes are distinguished: well differentiated, atypical and adenocarcinoid. Well differentiated tumors consist of goblet cells; however cuboidal or columnar cells may also occur. They infiltrate into the stroma or are located within the lakes of mucus. Atypical mucous carcinoid is formed by glandular, sieve or fine-cystic structures with moderate nuclear atypia. Histological structure of the adenocarcinoid is mixed of carcinoid and adenocarcinoma. Stromal carcinoid is compound of normal thyroid tissue and carcinoid, mostly trabecular type, rarely insular or mucinous.

Ovarian carcinoids are diagnosed in early clinical stage. Modlin et al. [18] analysed 113 cases of TC and found that 

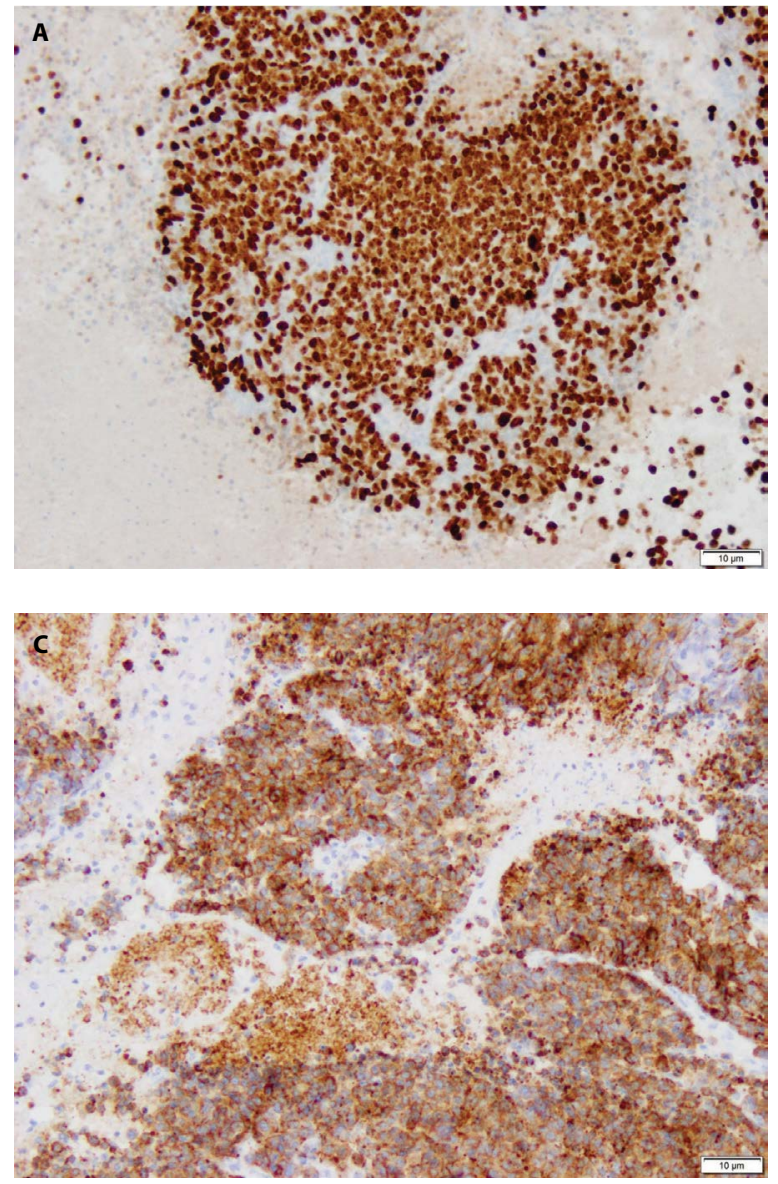

$66 \%$ of these tumors are locally advanced. Patients diagnosed with TC have good prognosis. Survival in stage I is more than $90 \%$, but in advanced stages does not exceed $33 \%$. Carcinoid metastasis from gastrointestinal tract or lungs is mandatory. Implants outside the ovary, bilateral tumors, vessels infiltrations and the absence of teratoma elements indicate a metastatic character. Sometimes these tumors can be misdiagnosed as Brenner tumors, granulosa cell tumor or Sertoli-Leydig cells tumors. Surgery is the treatment of choice. Fertility sparing surgery is possible in patients with normal contralateral ovary and Ki-67 index does not exceed $5 \%$.

\section{HG NENS}

These tumors are characterized by high mitotic activity and Ki-67 index usually above $30 \%$. These features determine aggressive clinical course of HG ovarian NEN.

Small cell carcinoma, hypercalcemic type (Fig. 3) is an exceedingly rare and lethal tumor, mostly affecting young women (the average age is 24 years) with aggressive clinical course. It develops usually unilaterally; bilateral or hereditary forms are rare. The prognosis is extremely unfavorable. An advanced tumor with multiple metastases is found in about $50 \%$ of cases at the time of diagnosis. Symptoms

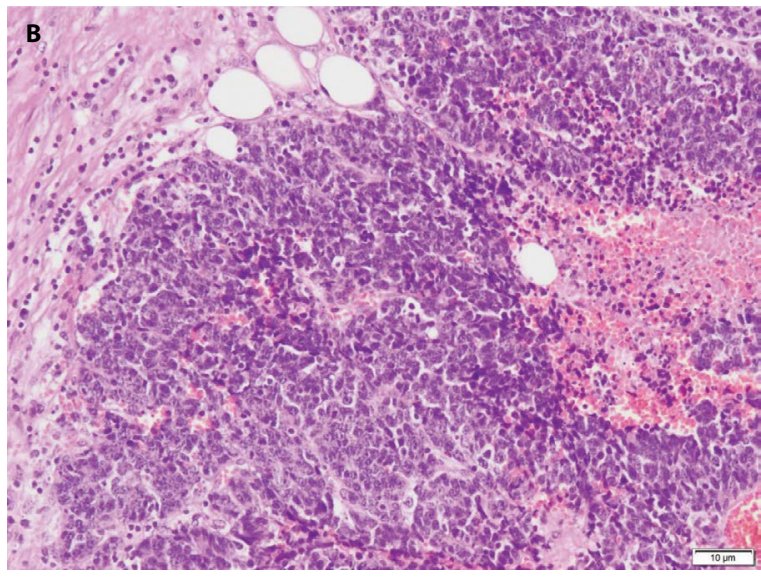

Figure 3. Small cell carcinoma, hypercalcemic type

A. Hematoxylin\&eosin staning; magnification $\times 100$. B. IHC. Positive synaptophysin reaction; magnification $\times 100$. C. IHC. Positive Ki-67 reaction in $100 \%$ cells; magnification $\times 100$

of paraneoplastic hypercalcemia occur in $2 / 3$ patients. This tumor can be misdiagnosed as granuloma, high grade ovarian cancer, dysgerminoma or other undifferentiated cancer. Kupryjańczyk et al. [26] described somatic and germinal mutations within the SMARCA4 gene, which causes a lack of BRG 1 protein. This may be helpful in differentiating from other tumors.

In small cell carcinoma, pulmonary type, metastasis from primary pulmonary malignancy always must be excluded. It is extremely rare aggressive ovarian tumor with an incidence of $<1 \%$, usually occurring in perimenopausal or postmenopausal women (the average age is 59 years). About $50 \%$ of cases occur bilaterally. It is diagnosed in advanced stages. Prognosis is poor. Patients sometimes demonstrate Cushing's syndrome-like symptoms.

Ovarian large cell carcinoma usually presents as unilateral lesion. An average age at the time of diagnosis is 48.5. It is a very rare form of cancer with an extremely aggressive clinical course. This neoplasm may co-exist with epithelial ovarian tumors such as mucinous borderline tumor, mucinous or serous cancers [27]. Symptoms and treatment of the disease are like these of ovarian cancer. The optimal surgery requires removal of all macroscopically lesions. An adjuvant chemotherapy is based on the regimens with cisplatin 
and etoposide and minimum five cycles are recommended $[23,25,27]$. Treatment with somatostatin analogues can be considered in tumors with $\mathrm{Ki}-67<30 \%$ and SST receptors revealed in scintigraphy. In HG NETs with Ki-67 > 30\% systemic treatment with cytotoxic drugs is mandatory.

\section{SUMMARY}

Neuroendocrine tumors of the cervix and ovaries are rare and require attentive histopathological diagnosis with immunohistochemical staining. The diagnosis should be always confirmed with chromogranin A and synaptophysin. The Ki-67 proliferation index is not always predictive or prognostic as in gastrointestinal neuroendocrine tumors. Ovarian carcinoids require differential diagnosis between primary ovarian cancers and metastases to ovaries, especially from the digestive system and pulmonary neoplasms. Treatment should be conducted by the most experienced oncology centers.

\section{Conflicts of interest}

The authors declare no conflict of interest.

\section{REFERENCES}

1. Chun YiK. Neuroendocrine Tumors of the Female Reproductive Tract: A Literature Review. J Pathol Transl Med. 2015 [Epub ahead of print], doi: 10.4132/jptm.2015.09.20, indexed in Pubmed: 26459408.

2. Atienza-Amores M, Guerini-Rocco E, Soslow RA, et al. Small cell carcinoma of the gynecologic tract: a multifaceted spectrum of lesions. Gynecol Oncol. 2014; 134(2): 410-418, doi: 10.1016/j.ygyno.2014.05.017, indexed in Pubmed: 24875120.

3. Solcia E, Klöppel G, Sobin LH. Histological typing of endocrine tumours. Science \& Business Media. 2012.

4. Kurman RJ, Carcangiu ML, Herrington CS. WHO classification of tumours of the female reproductive organs. Lyon IARC Press 2014.

5. Chiang YC, Chen YY, Hsieh SF, et al. Taiwan Cervical Cancer Prevention Surveillance Center. Screening frequency and histologic type influence the efficacy of cervical cancer screening: A nationwide cohort study. Taiwan J Obstet Gynecol. 2017; 56(4): 442-448, doi: 10.1016/j. tjog.2017.01.010, indexed in Pubmed: 28805598.

6. Li J, Ouyang Yi, Tao Y, et al. Small cell carcinoma of the cervix: analysis of clinical and pathological findings. Eur J Gynaecol Oncol. 1998; 19(2): 189-192, indexed in Pubmed: 9611065.

7. Zhou C, Hayes MM, Clement PB, et al. Small cell carcinoma of the uterine cervix: cytologic findings in 13 cases. Cancer. 1998; 84(5): 281-288, indexed in Pubmed: 9801202.

8. Park HJ, Choi YMi, Chung CK, et al. Pap smear screening for small cell carcinoma of the uterine cervix: a case series and review of the literature. J Gynecol Oncol. 2011; 22(1): 39-43, doi: 10.3802/jgo.2011.22.1.39, indexed in Pubmed: 21607094.

9. Kim MJ, Kim NaR, Cho HY, et al. Differential diagnostic features of small cell carcinoma in the uterine cervix. Diagn Cytopathol. 2008; 36(9): 618-623, doi: 10.1002/dc.20875, indexed in Pubmed: 18677758.

10. Tempfer CB, TischoffI, Dogan A, et al. Neuroendocrine carcinoma of the cervix: a systematic review of the literature. BMC Cancer. 2018; 18(1): 530, doi: 10.1186/s12885-018-4447-x, indexed in Pubmed: 29728073.

11. Kuji $S$, Watanabe $R$, Sato $Y$, et al. A new marker, insulinoma-associated protein 1 (INSM1), for high-grade neuroendocrine carcinoma of the ute- rine cervix: Analysis of 37 cases. Gynecol Oncol. 2017; 144(2): 384-390, doi: 10.1016/j.ygyno.2016.11.020, indexed in Pubmed: 27908529.

12. McCluggageWG, Kennedy K, Busam KJ. An immunohistochemical study of cervical neuroendocrine carcinomas: Neoplasms that are commonly TTF1 positive and which may express CK20 and P63. Am J Surg Pathol. 2010; 34(4): 525-532, doi: 10.1097/PAS.0b013e3181d1d457, indexed in Pubmed: 20182342.

13. Castle PE, Pierz A, Stoler MH. A systematic review and meta-analysis on the attribution of human papillomavirus (HPV) in neuroendocrine cancers of the cervix. Gynecol Oncol. 2018; 148(2): 422-429, doi: 10.1016/j. ygyno.2017.12.001, indexed in Pubmed: 29248196.

14. Fischerova D, Cibula D, Stenhova $H$, et al. Transrectal ultrasound and magnetic resonance imaging in staging of early cervical cancer. Int J Gynecol Cancer. 2008; 18(4): 766-772, doi: 10.1111/j.1525-1438.2007.01072.x, indexed in Pubmed: 17892456.

15. Parikh JH, Barton DPJ, Ind TEJ, et al. MR imaging features of vaginal malignancies. Radiographics. 2008; 28(1): 49-63; quiz 322, doi: 10.1148/rg.281075065, indexed in Pubmed: 18203930.

16. Gardner GJ, Reidy-Lagunes D, Gehrig PA. Neuroendocrine tumors of the gynecologic tract: A Society of Gynecologic Oncology (SGO) clinical document. Gynecol Oncol. 2011; 122(1): 190-198, doi: 10.1016/j. ygyno.2011.04.011, indexed in Pubmed: 21621706.

17. Lopes Dias J, Cunha TM, Gomes FV, et al. Neuroendocrine tumours of the female genital tract: a case-based imaging review with pathological correlation. Insights Imaging. 2015; 6(1): 43-52, doi: 10.1007/s13244014-0378-5, indexed in Pubmed: 25592289.

18. Modlin IM, Shapiro MD, Kidd M. An analysis of rare carcinoid tumors: clarifying these clinical conundrums. World J Surg. 2005; 29(1): 92-101, doi: 10.1007/s00268-004-7443-z, indexed in Pubmed: 15599742.

19. Gadducci A, Carinelli S, Aletti G. Neuroendrocrine tumors of the uterine cervix: A therapeutic challenge for gynecologic oncologists. Gynecol Oncol. 2017; 144(3):637-646, doi: 10.1016/j.ygyno.2016.12.003, indexed in Pubmed: 28057354.

20. Zivanovic O, Leitao MM, Park KJ, et al. Small cell neuroendocrine carcinoma of the cervix: Analysis of outcome, recurrence pattern and the impact of platinum-based combination chemotherapy. Gynecol Oncol. 2009; 112(3): 590-593, doi: 10.1016/j.ygyno.2008.11.010, indexed in Pubmed: 19110302.

21. Gibbs J, Mei S, Economos K, et al. Clinicopathologic features, incidence, and survival trends of gynecologic neuroendocrine tumors: a SEER database analysis. AJOG. 2019; 221(1): 53.e1-53.e6, doi: 10.1016/j. ajog.2019.02.052.

22. Chen J, Macdonald OK, Gaffney DK. Incidence, mortality, and prognostic factors of small cell carcinoma of the cervix. Obstet Gynecol. 2008; 111(6): 1394-1402, doi: 10.1097/AOG.0b013e318173570b, indexed in Pubmed: 18515524

23. Embry JR, Kelly MG, Post MD, et al. Large cell neuroendocrine carcinoma of the cervix: prognostic factors and survival advantage with platinum chemotherapy. Gynecol Oncol. 2011; 120(3): 444-448, doi: 10.1016/j. ygyno.2010.11.007, indexed in Pubmed: 21138780.

24. Salvo G, Gonzalez Martin A, Gonzales NR, et al. Updates and management algorithm for neuroendocrine tumors of the uterine cervix. Int J Gynecol Cancer. 2019; 29(6): 986-995, doi: 10.1136/ijgc-2019-000504, indexed in Pubmed: 31263021.

25. Sehouli J, Woopen H, Paaka M, et al. Neuroendocrine neoplasms of the ovary: a retrospective study of the North Eastern German Society of Gynecologic Oncology (NOGGO). Anticancer Res. 2016; 36: 1003-1010.

26. Kupryjańczyk J, Dansonka-Mieszkowska A, Moes-Sosnowska J, et al. Ovarian small cell carcinoma of hypercalcemic type- evidence of germline origin and SMARCA4 gene inactivation. a pilot study. Pol J Pathol. 2013;64(4): 238-246, doi: 10.5114/pjp.2013.39331, indexed in Pubmed: 24375037.

27. Yang X, Chen J, Dong R. Pathological features, clinical presentations and prognostic factors of ovarian large cell neuroendocrine carcinoma: a case report and review of published literature. J Ovarian Res. 2019; 12(1): 69, doi: 10.1186/s13048-019-0543-z, indexed in Pubmed: 31345245 . 